\title{
Properties of structure functions from helicity components of light quarks and antiquarks in the statistical model
}

\author{
Claude Bourrely* \\ Aix-Marseille Université, Université de Toulon, CNRS, CPT, Marseille, France
}

(Received 1 June 2018; revised manuscript received 21 September 2018; published 26 November 2018)

\begin{abstract}
In the quantum statistical parton distribution approach proposed more than one decade ago to describe the parton structure, new properties are now understood; in particular the relation between quarks and antiquarks leads to very specific properties. The simultaneous treatment of unpolarized and polarized parton distribution functions (PDFs) allows a determination of thermodynamical potentials (the master parameters of the model) which drive their behavior and consequently the behavior of the structure functions. The existence of a possible relation between the gluon and a $q \bar{q}$ pair leads to define a toy model for the unpolarized and polarized gluons. In view of forthcoming experimental results in the large- $x$ region, specific predictions made by the model are presented.
\end{abstract}

DOI: 10.1103/PhysRevC.98.055202

\section{INTRODUCTION}

The main objective is to build a quark structure where constitutive elements can be understood through their parameters, which are easily associated with the quark properties. The first point to clarify is the choice of a statistical model. Taking the example of a proton at rest, which contains three quarks, a statistical treatment seems not justified due to the low number of elements. However, when a proton is accelerated in a collider the energy increase has not only an effect on the mass but also to creates a large number of $q \bar{q}$ pairs or a quark-gluon plasma, which in a $p-p$ collison materializes mainly in primary unstable particles observed in a detector as a large number of tracks. The production of numerous pairs and gluons provide a justification for a statistical treatment of the parton interaction process. Moreover, the fact that a quark is described in the model by a Fermi function means that it is already dressed to live in a surrounding nuclear medium made of quarks.

The statistical approach is characterized by thermodynamical potentials whose values are the master parameters; they drive not only the shape of the parton distribution functions (PDFs) but are found to control some specific properties of the structure functions. In order to introduce the maximum constraints, we decided to work from the beginning with helicity components, which are the building blocks of both the polarized and unpolarized PDFs; a unique situation in the domain. It is clear that the polarized data set is much

*claude.bourrely@cpt.univ-mrs.fr

Published by the American Physical Society under the terms of the Creative Commons Attribution 4.0 International license. Further distribution of this work must maintain attribution to the author(s) and the published article's title, journal citation, and DOI. Funded by $S C O A P^{3}$. smaller than the unpolarized one, and also that it is limited to a medium energy region; however, the experiments at the BNL Relativistic Heavy Ion Collider (RHIC) enlarge somehow this domain, but a large gap remains to reach energies available at the CERN Large Hadron Collider (LHC).

The objective of the paper is to discuss the consequence of the statistical approach on the quark structure, because from a collection of results obtained through the years one gets now a better global view.

In Sec. II, a review of the basic elements of the quarks distribution is presented together with the notations. In Sec. III, a proof is given to show how the antiquark PDFs can be deduced from those of the quarks by using different constraints in the fitting process. Section IV is devoted to an analysis of the different helicity components and how their effect is put in evidence in structure functions. In Sec. V, a toy model is introduced to define new unpolarized and polarized gluon PDFs, which is inspired by the relation between gluons and $q \bar{q}$ pairs. The conclusions are presented in Sec. VI.

\section{BASIC ELEMENTS OF THE QUARK DISTRIBUTIONS}

The PDFs are the essential elements to evaluate scattering processes in QCD. In the absence of a theory they are usually parametrized with polynomials [1,2]. To go beyond this approximation and in an attempt to define a more physical structure for the quarks, a statistical approach was proposed many years ago to build up the PDFs [3].

Let us now describe the main features of the statistical approach. The fermion distributions are given by the sum of two terms, a quasi Fermi-Dirac function of helicity $h= \pm$ and a helicity-independent diffractive contribution:

$$
x q^{h}\left(x, Q_{0}^{2}\right)=\frac{A_{q} X_{q}^{h} x^{b_{q}}}{\exp \left[\left(x-X_{q}^{h}\right) / \bar{x}\right]+1}+\frac{\tilde{A}_{q} x^{\tilde{b}_{q}}}{\exp (x / \bar{x})+1}
$$


for the quarks, and for the antiquarks the ansatz

$$
x \bar{q}^{h}\left(x, Q_{0}^{2}\right)=\frac{\bar{A}_{q}\left(X_{0 q}^{-h}\right)^{-1} x^{\bar{b}_{q}}}{\exp \left[\left(x+X_{0 q}^{-h}\right) / \bar{x}\right]+1}+\frac{\tilde{A}_{q} x^{\tilde{b}_{q}}}{\exp (x / \bar{x})+1},
$$

at the input energy scale $Q_{0}^{2}=1 \mathrm{GeV}^{2}$.

With the above definitions, the diffractive term is the same for flavors $u, d$, but has a specific expression for other flavors. It is absent in the quark helicity distribution $\Delta q=q^{+}$ $q^{-}$, the quark valence contribution $q-\bar{q}$, and the difference $u-d$.

In the numerator of the non-diffractive parts of Eq. (1), the multiplicative factor $X_{q}^{h}$ allows one to separate $u$ and $d$ quarks since one assumes $A_{u}=A_{d}$, the term $x^{b_{q}}$ implying a modification of the quantum statistical form; this term is introduced in order to control the small- $x$ behavior. The parameter $\bar{x}=0.09$ plays the role of a universal temperature and $X_{q}^{ \pm}$ are the two thermodynamical potentials of a quark $q$, with helicity $h= \pm$. They represent the fundamental parameters of the model because they drive the PDFs' behavior. ${ }^{1}$ For convenience the values of the potentials obtained in BS15 [5] are recalled:

$$
\begin{gathered}
X_{u}^{+}=0.475 \pm 0.001, \quad X_{u}^{-}=0.307 \pm 0.001, \\
X_{d}^{+}=0.245 \pm 0.001, \quad X_{d}^{-}=0.309 \pm 0.001, \\
X_{s}^{+}=0.011 \pm 0.001, \quad X_{s}^{-}=0.015 \pm 0.001 .
\end{gathered}
$$

\section{GENERATION OF THE ANTIQUARK DISTRIBUTIONS}

To adopt a coherent scheme, it is natural to suppose that antiquarks must also contain a Fermi part analogous to the quarks and, in addition, a diffractive part that is the same as in the quarks. All these constraints lead to a general expression,

$$
x \bar{q}^{h}\left(x, Q_{0}^{2}\right)=\frac{\bar{A}_{q}^{\prime h} x^{\bar{b}_{q}}}{\exp \left[\left(x-Y_{q}^{h}\right) / \bar{x}\right]+1}+\frac{\tilde{A}_{q} x^{\tilde{b}_{q}}}{\exp (x / \bar{x})+1} .
$$

This distribution depends on the new parameters $\bar{A}_{q}^{\prime h}, Y_{q}^{h}$, compared to Eq. (2). In order to determine these parameters in a fitting process, the constraint of the valence sum rule is added,

$$
\int[q(x)-\bar{q}(x)] d x=N_{q}, \quad \text { where } N_{q}=2,1 \text { for } u, d
$$

(this sum rule is independent of the diffractive part). A second constraint, which comes from the momentum sum rule, is added:

$$
\int \sum_{i}\left[x q_{i}(x)+x \bar{q}_{i}(x)+x G(x)\right] d x=1,
$$

where $G(x)$ is the unpolarized gluon distribution. Making a fit at next-to-leading order (NLO) of unpolarized and polarized experimental data analogous to the one discussed in BS15 [5],

\footnotetext{
${ }^{1}$ The PDF QCD evolution was done at next-to-leading order in the $\overline{\mathrm{MS}}$ scheme using the HOPPET program [4].
}

one finds for the potentials a solution

$$
\begin{array}{ll}
Y_{u}^{-}=-0.475, & Y_{u}^{+}=-0.307, \\
Y_{d}^{-}=-0.244, & Y_{d}^{+}=-0.309,
\end{array}
$$

where a comparison with the solution obtained in BS15 (3) leads to

$$
Y_{u}^{-}=-X_{u}^{+}, Y_{u}^{+}=-X_{u}^{-}, Y_{d}^{-}=-X_{d}^{+}, Y_{d}^{+}=-X_{d}^{-},
$$

the change of sign in the $\bar{q}$ potentials and in the helicity find its origin from the unpolarized gluon whose potential is null, $X_{q}^{ \pm}+Y_{q}^{\mp}=0$; this point will be examined later.

The other parameters are given by

$$
\begin{aligned}
A & =1.943, \quad b_{u}=b_{d}=0.471, \quad \bar{b}_{u}=\bar{b}_{d}=1.304, \\
\bar{A}_{u}^{\prime+} & =29.039, \quad \bar{A}_{u}^{\prime-}=18.768, \\
\bar{A}_{d}^{\prime+} & =28.851, \quad \bar{A}_{d}^{\prime-}=36.536 .
\end{aligned}
$$

By introducing the definition $\bar{A}_{q}^{\prime h}=\bar{A}_{q} / X_{q}^{-h}$, the antiquark distributions (4) become identical to Eq. (2), where the four normalizations $\bar{A}_{q}^{\prime} h$ are reduced to one constant, $\bar{A}_{q}=8.915$. This result confirms the ansatz intrduced at the beginning for the antiquarks, which was expected to be a solution of Eq. (4). To summarize, an interesting relation between light quarks and antiquarks in the statistical approach was established, with the objective of reducing the number of arbitrary distributions (see Sec. V).

\section{PROPERTIES OF THE UNPOLARIZED AND POLARIZED QUARK DISTRIBUTIONS}

From the results obtained in Eq. (3) one finds for the light quarks the following hierarchy between the different potential components:

$$
X_{u}^{+}>X_{u}^{-} \simeq X_{d}^{-}>X_{d}^{+} .
$$

In Eq. (7) the two potentials $X_{u}^{-}, X_{d}^{-}$have very close numerical values, which is a consequence of the near equality between $x u^{-}\left(x, Q^{2}\right)$ and $x d^{-}\left(x, Q^{2}\right)$.

It is easy to show that quark helicity PDFs increase with the potential values, while for antiquarks helicity PDFs increase when the potentials decrease.

As a consequence of the above hierarchy of potentials (10), there follows a hierarchy on the quarks helicity distributions,

$$
x u_{+}(x)>x u_{-}(x)=x d_{-}(x)>x d_{+}(x)
$$

and an obvious hierarchy for the antiquarks, namely

$$
x \bar{d}_{-}(x)>x \bar{d}_{+}(x)=x \bar{u}_{+}(x)>x \bar{u}_{-}(x) .
$$

It is important to note that these inequalities (11) and (12) are preserved by the NLO QCD evolution. Also note that the initial analytic form, Eqs. (1) and (2), is almost preserved by the $Q^{2}$ evolution with some small changes on the parameters' numerical values. One clearly concludes that $u\left(x, Q^{2}\right)>d\left(x, Q^{2}\right)$ implies a flavor-asymmetric light sea, i.e., $\bar{d}\left(x, Q^{2}\right)>\bar{u}\left(x, Q^{2}\right)$, a trivial consequence of the Pauli exclusion principle, which is built in. Indeed this is based on the fact that the proton contains two $u$ quarks and only one $d$ quark. 
We move on to mention more significant consequences concerning the helicity distributions that follow from Eqs. (7)-(12). First for the $u$ quark

$$
x \Delta u\left(x, Q^{2}\right)>0, \quad x \Delta \bar{u}\left(x, Q^{2}\right)>0 .
$$

Similarly for the $d$ quark

$$
x \Delta d\left(x, Q^{2}\right)<0, \quad x \Delta \bar{d}\left(x, Q^{2}\right)<0 .
$$

These predictions were made almost 15 years ago [3]. It is interesting to notice that the polarized structure function $x g_{1}^{p}$ measured by experiment and driven by $x \Delta u$ has a maximum around $x=0.42$ in a medium $Q^{2}$ range; such an $x$ value is close to the thermodynamical potential $X_{u}^{+}$. Concerning $x g_{1}^{n}$, which is negative for small $x$ because it is dominated by $x \Delta d$, when $x$ increases $x \Delta u$ becomes dominant so $x g_{1}^{n}$ takes positive values. All these properties are well understood and described by the statistical model due to the properties of thermodynamical potentials. The predicted signs and magnitudes have also been confirmed [5] by the measured single-helicity asymmetry $A_{L}$ in the $W^{ \pm}$production at BNL-RHIC from the STAR experiment [6].

Another important earlier prediction concerns the deep inelastic scattering (DIS) asymmetries, more precisely $\left[\Delta u\left(x, Q^{2}\right)+\Delta \bar{u}\left(x, Q^{2}\right)\right] /\left[\left(u\left(x, Q^{2}+\bar{u}\left(x, Q^{2}\right)\right] \quad\right.\right.$ and $\left[\Delta d\left(x, Q^{2}\right)+\Delta \bar{d}\left(x, Q^{2}\right)\right] /\left[\left(d\left(x, Q^{2}\right)+\bar{d}\left(x, Q^{2}\right)\right]\right.$, shown in Fig. 1. Note that the data from the HERMES experiment [7-9] and the Jefferson Lab (Jlab) [10,11], so far, are in agreement with these predictions at low $x<0.6$. In the high- $x$ region our prediction differs from those which impose, for both quantities, the value 1 for $x=1$. This is another challenge, since they have been measured at JLab $[10,11]$ only up to $x=0.6$.

There are two more important consequences which relate unpolarized and helicity distributions. Namely, for quarks

$x u\left(x, Q^{2}\right)-x d\left(x, Q^{2}\right)=x \Delta u\left(x, Q^{2}\right)-x \Delta d\left(x, Q^{2}\right)>0$,

and similarly for antiquarks

$$
x \bar{d}\left(x, Q^{2}\right)-x \bar{u}\left(x, Q^{2}\right)=x \Delta \bar{u}\left(x, Q^{2}\right)-x \Delta \bar{d}\left(x, Q^{2}\right)>0 .
$$

This means that the flavor asymmetry of the light antiquark distributions is the same for the corresponding helicity distributions, as noticed a long time ago [12] (see also Ref. [13]).

Now coming back to all the components $x u_{+}\left(x, Q^{2}\right), \ldots, x \bar{u}_{-}\left(x, Q^{2}\right)$ and more precisely to their $x$ behavior. It is clear that $x u_{+}\left(x, Q^{2}\right)$ is the largest one and they are all monotonic decreasing functions of $x$ at least for $x>0.2$, outside the region dominated by the diffractive contribution.

Similarly $x \bar{d}_{-}\left(x Q^{2}\right)$ is the largest of the antiquark components. Therefore if one considers the ratio $d\left(x, Q^{2}\right) / u\left(x, Q^{2}\right)$, its value is 1 at $x=0$, because the diffractive contribution dominates and, due to the monotonic-decrease property, it decreases for an increasing $x$. This falling $x$ behavior has been verified experimentally from the ratio of the DIS structure functions $F_{2}^{d} / F_{2}^{p}$ and the charge asymmetry of the $W^{ \pm}$ production in $\bar{p} p$ collisions [14].

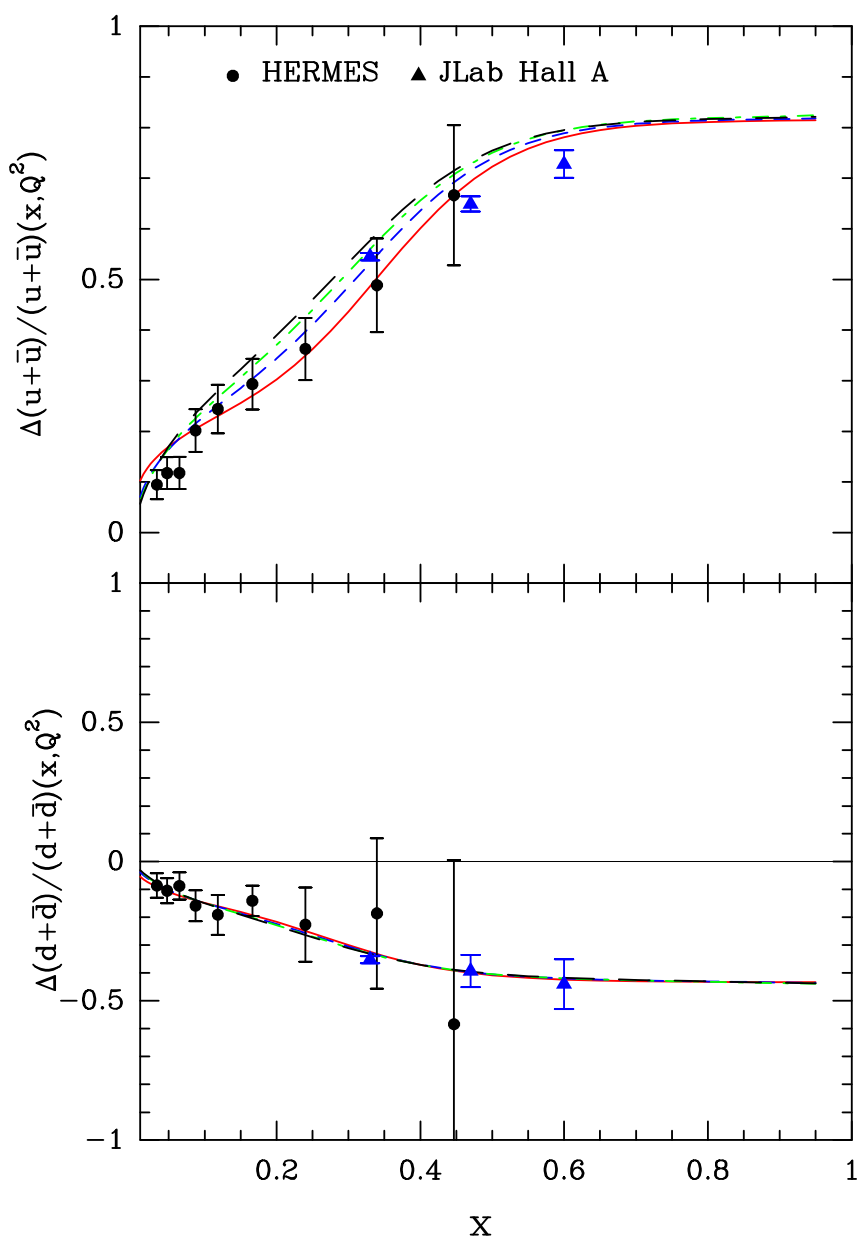

FIG. 1. BS15 [5] predicted ratios $\left[\Delta u\left(x, Q^{2}\right)+\Delta \bar{u}\left(x, Q^{2}\right)\right] /$ $\left[u\left(x, Q^{2}+\bar{u}\left(x, Q^{2}\right)\right]\right.$ and $\left[\Delta d\left(x, Q^{2}\right)+\Delta \bar{d}\left(x, Q^{2}\right)\right] /\left[\left(d\left(x, Q^{2}+\right.\right.\right.$ $\left.\bar{d}\left(x, Q^{2}\right)\right]$ versus $x$, at $Q^{2}=1$ (solid), 10 (dashed), 100 (dasheddotted), and $1000 \mathrm{GeV}^{2}$ (long-dashed). Experiments data are from the HERMES [7-9] and Jlab [10,11] Collaborations.

Similarly if one considers the ratio $\bar{u}\left(x, Q^{2}\right) / \bar{d}\left(x, Q^{2}\right)$, its value is 1 at $x=0$, because the diffractive contribution dominates and, due to the monotonic-decrease property, it also decreases for an increasing $x$. By looking at the curves of Fig. 2, one sees similar behaviors. In both cases in the vicinity of $x=0$ one has a sharp behavior due to the fact that the diffractive contribution dominates, and in the high $x$ region there is a flattening out above $x \simeq 0.6$. It is remarkable to observe that these ratios have almost no $Q^{2}$ dependence.

To conclude, we predict a monotonic increase of the ratio $\bar{d}\left(x, Q^{2}\right) / \bar{u}\left(x, Q^{2}\right)$. This was first observed in the low- $x$ region by the E866/NuSea Collaboration $[15,16]$ and very recently there was a serious indication from the preliminary results of the SeaQuest Collaboration that this trend persists beyond $x=0.2[17]$.

\section{A TOY MODEL FOR GLUON DISTRIBUTIONS}

In the BS15 version of the model [5], the unpolarized gluon is parametrized as a Bose-Einstein function with a zero 

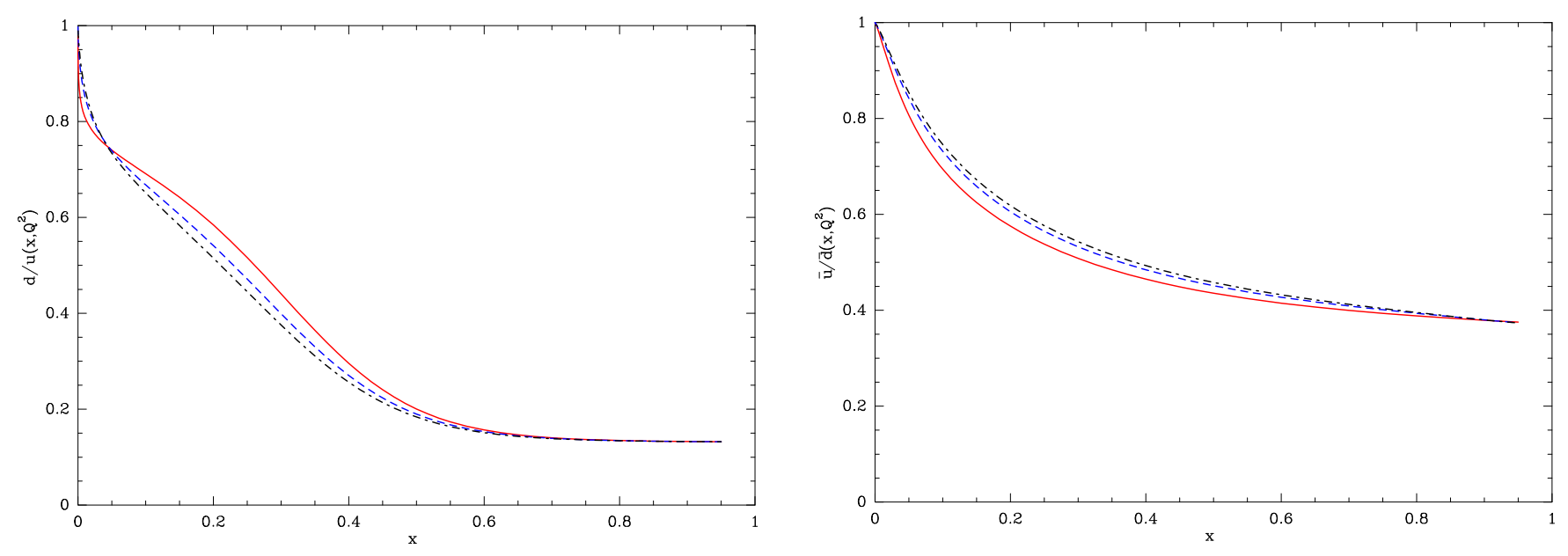

FIG. 2. The ratios $d\left(x, Q^{2}\right) / u\left(x, Q^{2}\right)$ (left) and $\bar{u}\left(x, Q^{2}\right) / \bar{d}\left(x, Q^{2}\right)$ (right) versus $x$ for $Q^{2}=1$ (solid), 10 (dashed), and $100 \mathrm{GeV}$ (dasheddotted), from BS15 [5].

potential value, and no diffractive part is included:

$$
x G\left(x, Q_{0}^{2}\right)=\frac{A_{G} x^{b_{G}}}{\exp (x / \bar{x})-1},
$$

where $A_{G}=36.778$ is determined by the momentum sum rule. The polarized gluon distribution involves also a BoseEinstein function but requires an extra factorized function whose origin is discussed in Refs. [18,19], so its expression is given by

$$
x \Delta G\left(x, Q_{0}^{2}\right)=\frac{\tilde{A}_{G} x^{\tilde{b}_{G}}}{\left(1+c_{G} x^{d_{G}}\right)} \frac{1}{\exp (x / \bar{x})-1},
$$

where $\tilde{A}_{G}=26.887$. In contrast to the quark situation, these expressions are not directly related and so have to be determined independently from specific experimental data. Coming back to the model structure, this is not exactly true because their determination is influenced by unpolarized and polarized quarks, which are related; nevertheless, a more direct relation will reinforce the model structure.

Inside a proton at high energy, besides the presence of $2 u+d$ quarks, there exists a collection of $q-\bar{q}$ pairs and gluons. It is also known that a quark-antiquark pair can annihilate into two gluons. It seems natural to suppose that a $q-\bar{q}$ pair should behave like a composite boson and so could have a relation with the gluon field. In this case one should find that in a QCD process involving gluons, for instance in structure functions, one can replace the gluon by a $q-\bar{q}$ pair, leading to a new test for the antiquarks since the quarks are well established.

For this purpose two new formulas are defined for the unpolarized and polarized gluons, and they play the role of a toy model at the input scale. In these formulas $q$ and $\bar{q}$ contain only the non-diffractive part of Eqs. (1) and (2), and, to comply with the previous definitions (17) and (18), their expressions are now given by

$$
x G\left(x, Q_{0}^{2}\right)=A_{q \bar{q}}(x u x \cdot x \bar{u}+x d \cdot x \bar{d}+x s \cdot x \bar{s})\left[x, Q_{0}^{2}\right],
$$

$$
\begin{aligned}
x \Delta G\left(x, Q_{0}^{2}\right)= & A_{\delta q \bar{q}}(x \Delta u \cdot x \Delta \bar{u}+x \Delta d \cdot x \Delta \bar{d} \\
& +x \Delta s \cdot x \Delta \bar{s})\left[x, Q_{0}^{2}\right] .
\end{aligned}
$$

We remark that although the two formulas (19) and (20) contain the product of two Fermi functions, both are evolved as a boson, so the result is not the evolution of the product of two Fermi distributions.

Also, in the expressions (17) and (18) $G$ and $\Delta G$ are defined independently and are not related, while in the expressions (19) and (20) indeed they are related, because for a given flavor $q, \bar{q}, \Delta q, \Delta \bar{q}$ are not independent. This has the consequence that the parton structure can be described with very few basic constituents.

A fit at NLO of unpolarized and polarized DIS experimental data gives in the case of BS15 parametrization [5]

$$
\chi^{2}=2860, \quad 2140 \mathrm{pts}, \quad 1.34 \chi^{2} / \mathrm{pt} .
$$

Now with Eqs. (19) and (20) of the toy model a fit of the same set of data gives

$$
\chi^{2}=3013, \quad 2140 \mathrm{pts}, \quad 1.4 \chi^{2} / \mathrm{pt} ;
$$

the difference in $\chi^{2}$ is $5 \%$. Restricted to the polarized structure functions $g_{1}^{p}, g_{1}^{d}, g_{1}^{n}$ with 271 pts, BS15 gives $\chi^{2}=323$ and the toy model gives $\chi^{2}=301$. Notice that in the original version the expression of $\Delta G$ requires four parameters; in this version only one normalization constant $A_{\delta q \bar{q}}$ is necessary, since $A_{G}, A_{q \bar{q}}$ are determined by the momentum sum rule. This difference confirms the interest in the gluon distributions given by Eqs. (19) and (20).

In this new fit the potentials read

$$
\begin{aligned}
X_{u}^{+} & =0.4616, \quad X_{u}^{-}=0.3166, \\
X_{d}^{+} & =0.2530, \quad X_{d}^{-}=0.3062, \\
X_{s}^{+} & =0.007896, \quad X_{s}^{-}=00982, \\
b_{q} & =0.491, \quad \bar{b}_{q}=1.123, \\
b_{s} & =0.0044, \quad \bar{b}_{s}=0.08, \\
\bar{x} & =0.0944 .
\end{aligned}
$$



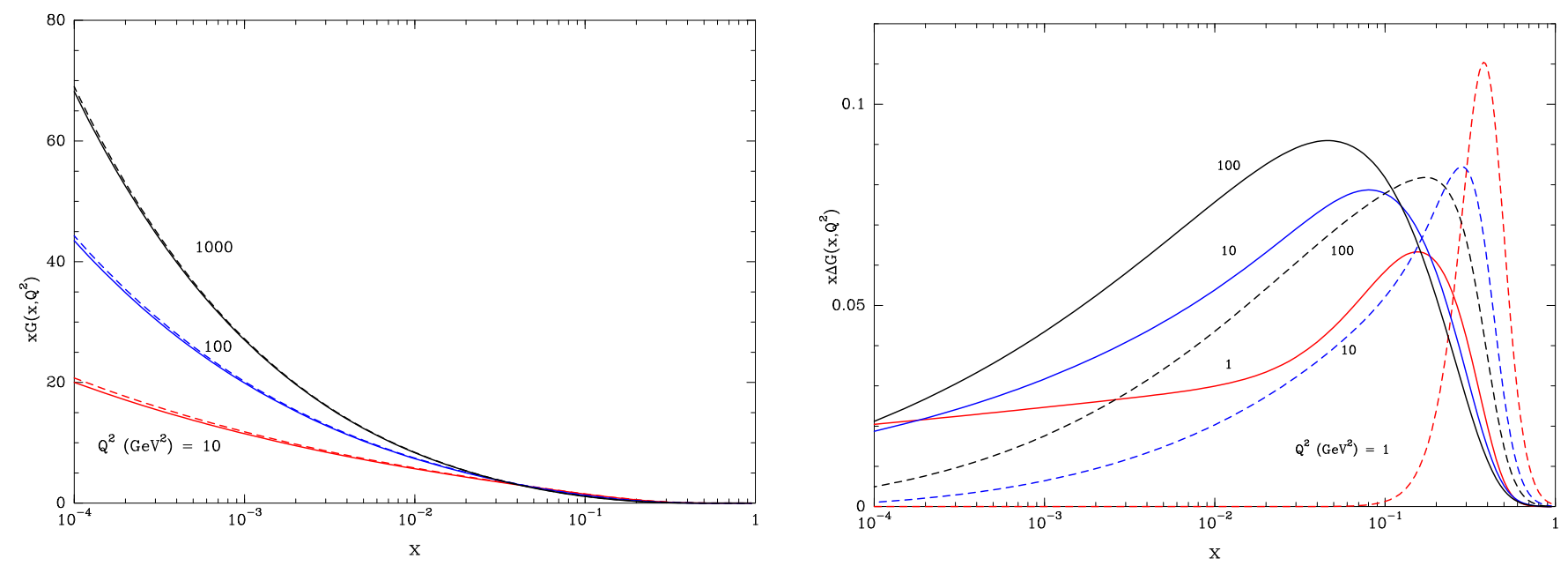

FIG. 3. Left: Comparison of $x G\left(x, Q^{2}\right)$ versus $x$ for $Q^{2}=1,10,100 \mathrm{GeV}^{2}$, computed from BS15 [5] (dashed) and the toy model (solid). Right: Same comparison but for $x \Delta G\left(x, Q^{2}\right)$.

The new results for the potential values are close to the previous ones (7) and still satisfy the previous hierarchy (10):

$$
X_{u}^{+}>X_{d}^{-} \sim X_{u}^{-}>X_{d}^{+},
$$

so the properties discussed in Sec. IV remain valid.

For the normalization constants one obtains $A_{q \bar{q}}=23.882$, $A_{\delta q \bar{q}}=18.99$.

In Fig. 3 a plot is given for some results associated with the unpolarized and polarized gluons in the cases of BS15 parametrization [(17) and (18)] (dashed curves) and the toy model [(19) and (20)] (solid curves). The distributions behaviors are very similar; the polarized case, which is more sensitive to the gluon structure, looks slightly different, but when combined with the polarized quarks give an excellent description of the polarized structure functions (see the $\chi^{2}$ values discussed above). To conclude this part devoted to the statistical model, the present formulas used as a toy parametrization of unpolarized and polarized gluons give an equivalent description of the original model, and they represent also a new test for the antiquark PDFs since the quark PDFs are well established. In QCD calculations Mellin transforms are sometime involved; the Mellin transforms of the Fermi functions for fermions and bosons are mathematically related [20], which is an encouraging sign for our new definition of gluons.

One can ask the question if the previous formulation can be applied to another model. In the domain of polarized PDFs, the de Florian-Sassot-Stratmann-Vogelsang (DSSV) model [21] is a reference, so it becomes of interest to test this polarized version inside the toy model, taking the DSSV model as input in Eq. (20). There is a difference between the statistical model and the DSSV model due to the fact that in the statistical model unpolarized and polarized PDFs are related, which is not the case with DSSV. Polarized quarks, antiquarks, and the gluon of flavor $i$ are defined in DSSV at the input scale $\mu_{0}$ by the expressions (28) of Ref. [21], namely

$$
x \Delta f_{i}\left(x, \mu_{0}^{2}\right)=N_{i} x^{\alpha_{i}}(1-x)^{\beta_{i}}\left(1+\gamma_{i} \sqrt{x}+\eta_{i} x\right) .
$$

A more serious constraint on the polarized gluon can be obtained from the double-spin asymmetry in jet production $A_{L L}^{\text {jet }}$ with the modified expression for the polarized DSSV gluon [22]

$$
x \Delta g\left(x, Q_{0}^{2}\right)=N_{g} x^{\alpha_{g}}(1-x)^{\beta_{g}}\left(1+\eta_{g} x^{\kappa_{g}}\right) .
$$

In order to test the toy model with the polarized gluon, Eq. (20), one adopts the strategy of fitting the same polarized data previously used, taking Eqs. (25) for the quarks and Eq. (20) for the polarized gluon. In Table I, for simplicity, the number of quarks free parameters is restricted to $N_{i}, \eta_{i}$, while $\alpha_{i}, \beta_{i}, \gamma_{i}$, are held fixed to their original values (see Table II of Ref. [21]).

For the polarized gluon one obtains a normalization coefficient $A_{\delta q \bar{q}}=-0.078$. With a $\chi^{2}=235$ for 271 pts the quality of the polarized fit is similar to the previous statistical model. Here again the five parameters introduced in Eq. (26) are reduced to one. A plot of the polarized gluon for three $Q^{2}$ values is shown in Fig. 4 for the original DSSV model (dashed curve) and the toy model (solid curve).

Now our purpose is to show that the polarized gluon discussed above offers a good exploratory domain for the parton structure. Beginning with the statistical model, it was natural to associate to the gluon a Bose-Einstein expression such that

$$
x \Delta G\left(x, Q_{0}^{2}\right)=\tilde{A}_{G} x^{\tilde{b}_{G}} \frac{1}{\exp (x / \bar{x})-1} .
$$

TABLE I. Parameters describing NLO ( $\overline{\mathrm{MS}}) x \Delta f_{i}$ in Eq. (25) at the input scale $\mu_{0}=1 \mathrm{GeV}$, using the toy model.

\begin{tabular}{lccccc}
\hline \hline Flavor $i$ & $N_{i}$ & $\alpha_{i}$ & $\beta_{i}$ & $\gamma_{i}$ & $\eta_{i}$ \\
\hline$u+\bar{u}$ & 0.403 & 0.692 & 3.34 & -2.18 & 21.38 \\
$d+\bar{d}$ & -0.023 & 0.164 & 3.89 & 22.40 & 83.80 \\
$\bar{u}$ & 4.83 & 0.692 & 10.0 & 0 & 24.97 \\
$\bar{d}$ & -0.147 & 0.164 & 10.0 & 0 & 98.94 \\
$s=\bar{s}$ & -0.019 & 0.164 & 10.0 & 0 & -23.03 \\
\hline \hline
\end{tabular}




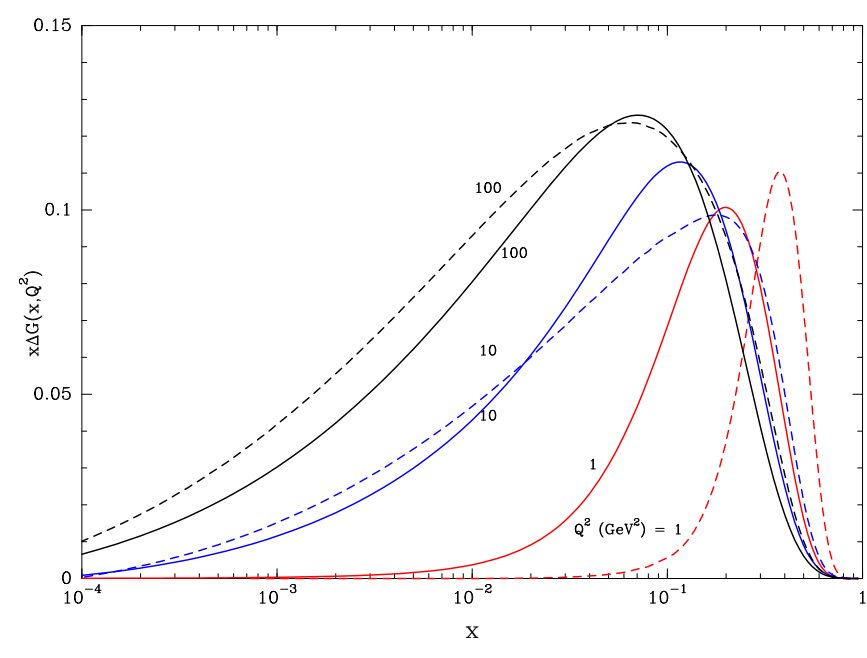

FIG. 4. Comparison of $x \Delta G\left(x, Q^{2}\right)$ versus $x$ for $Q^{2}=$ $1,10,100 \mathrm{GeV}^{2}$ calculated with the toy DSSV model (solid) and the original one (dashed).

This original expression was unable to describe the doublespin asymmetry of the one-jet inclusive production $A_{L L}^{\text {jet }}$ in the near forward rapidity region as a function of $p_{T}$ within the domain $5 \leqslant p_{T} \leqslant 30 \mathrm{GeV}$ measured by the STAR Collaboration at BNL-RHIC [23]. To obtain a good description the polarized gluon was modified according to Eq. (18).

It turns out that the extra multiplicative function $\frac{1}{\left(1+c_{G} x^{\left.d_{G}\right)}\right.}$ has the behavior of a logistic function or activation function used in neural networks [18],

$$
S(x)=\frac{1}{1+e^{-e_{G} x+h_{G}}},
$$

so one can write the polarized gluon as

$$
x \Delta G\left(x, Q_{0}^{2}\right)=S(x) \frac{\tilde{A}_{G}^{\prime} x^{b_{G}}}{\exp (x / \bar{x})-1} .
$$

The physical interpretation of this new formula means that the incoming momentum is collected now by means of a BoseEinstein distribution and then filtered by an activation function to produce the gluon probability distribution.

The toy model defined above proceeds along the same line: a polarized gluon is built in terms of a composite made of known physical functions, namely the PDFs associated with their probability. Figure 5 shows the example of $u, \bar{u}$ quarks where their probabilities product generates a component of the gluon polarized PDF. The resulting effect of the toy model is perfectly compatible with experimental data for both the statistical and DSSV models.

To summarize the discussion on the different expressions so far defined in (20) and (27)-(29), the objective was to replace an arbitrary function by a physical quantity perfectly justified in the context of the model.

It is known that $\Delta G$ gives an important contribution to the proton spin sum rule. A study of this effect is presented in Fig. 3 of Ref. [19] using the gluon defined by Eq. (18). It can be seen that just above $Q^{2}=100 \mathrm{GeV}^{2}$ the value of the spin sum rule $1 / 2$ is saturated; the same calculation performed

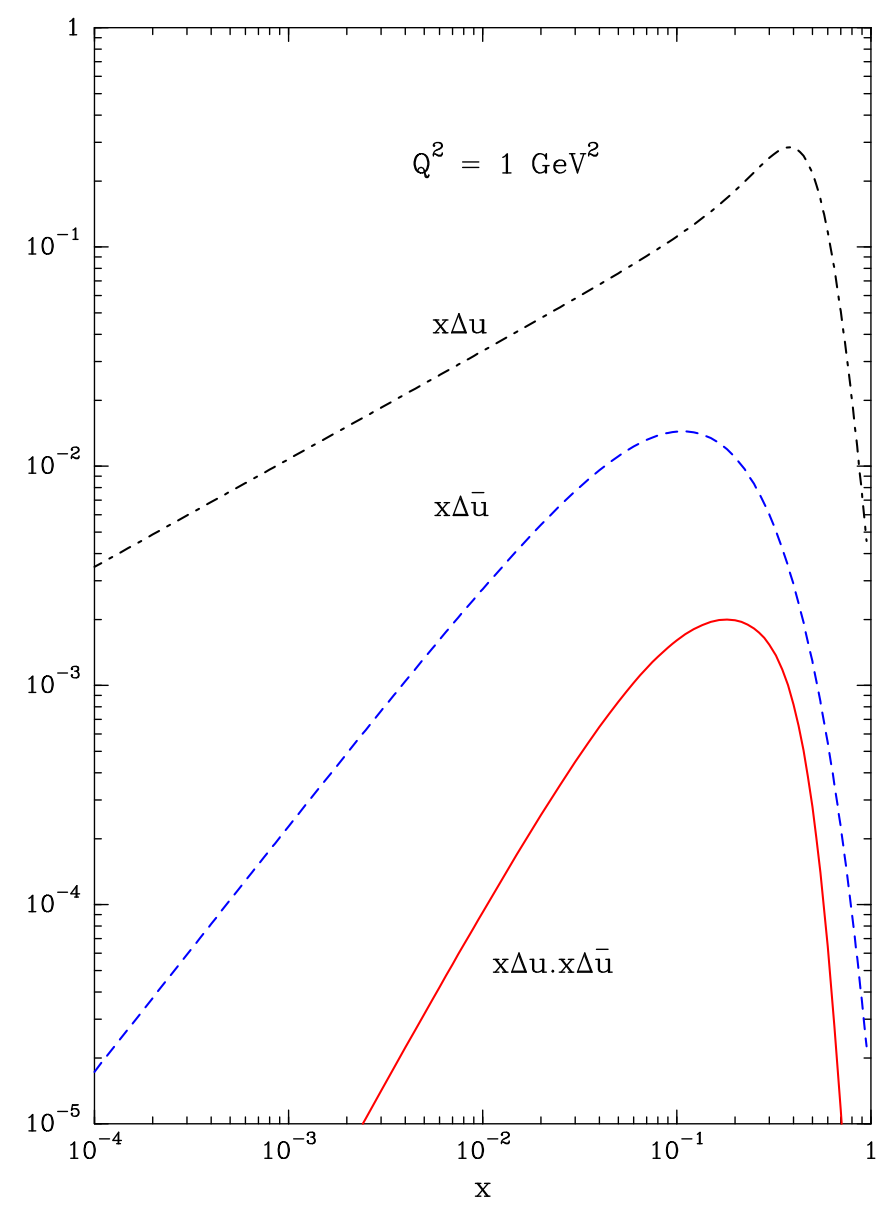

FIG. 5. Quark $u$ contribution to the polarized $\Delta G$ following the toy expression (20).

with the toy gluon model (20) gives a saturation for $Q^{2}$ around $1000 \mathrm{GeV}^{2}$, which corresponds to a significant improvement.

Finally, I would like to present a new test of the toy gluon distribution in a pure hadronic reaction and compute the double-helicity asymmetry $A_{L L}^{\mathrm{jet}}$ discussed above. It is important to remark that the asymmetry calculation requires knowledge of both the unpolarized and polarized gluon distributions (19) and (20). In Fig. 6 the prediction is compared with these high-statistics data points and the agreement is very reasonable.

\section{CONCLUSION}

The purpose of this work was to show that a statistical model offers a unique framework to build a quark structure whose properties are clearly defined by parameters related to physical quantities in the PDF expressions. The thermodynamical potentials which are the master parameters generate definite properties of the quark PDFs, as confirmed by experimental structure functions.

This prediction results from the following characteristic features of the statistical approach: 

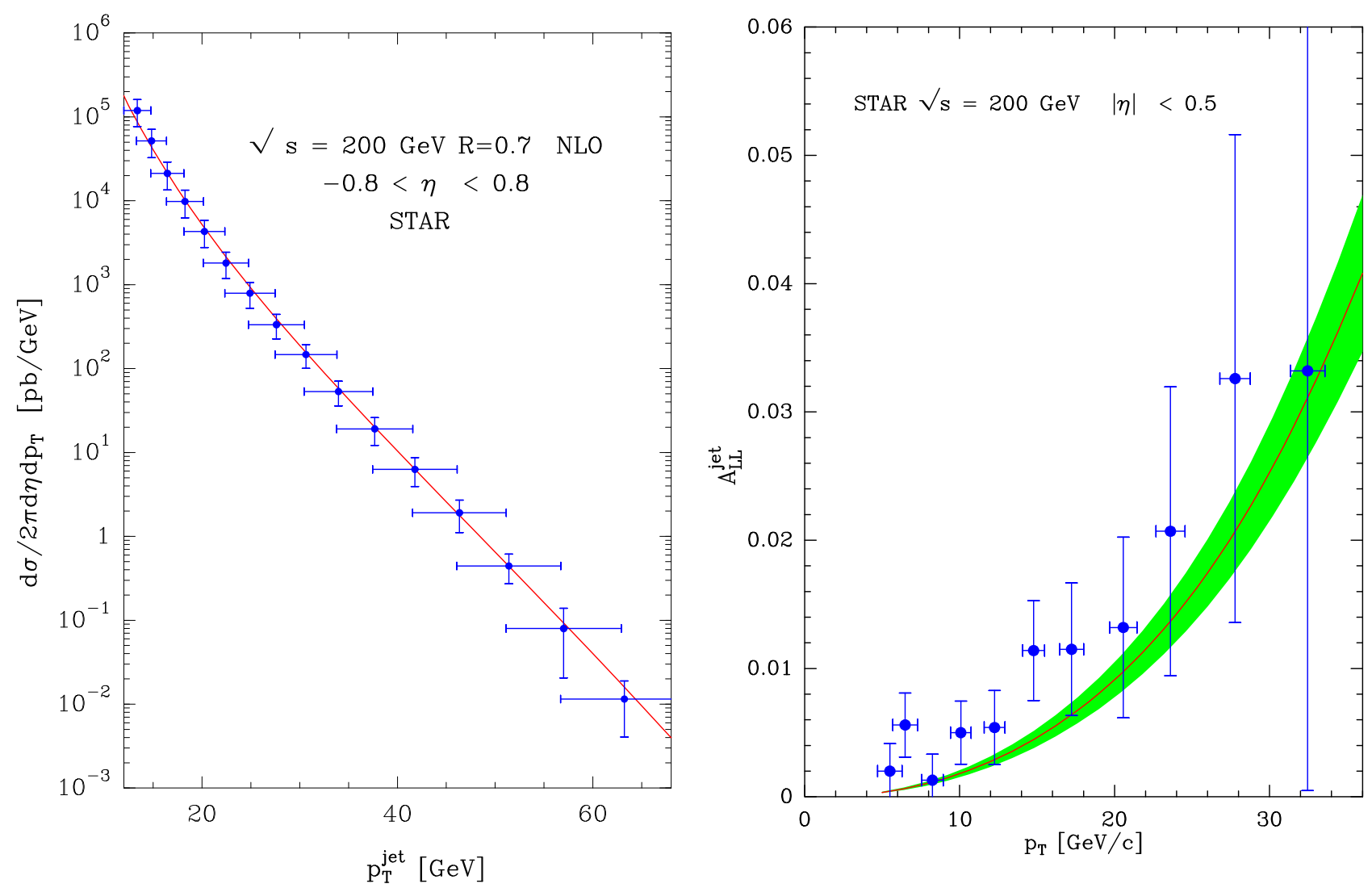

FIG. 6. Left: Inclusive differential cross section for $p+p \rightarrow$ jet $+X$ at $\sqrt{s}=200 \mathrm{GeV}$ versus jet $p_{T}$ calculated with the unpolarized toy gluon model (19). Right: The longitudinal double-spin asymmetry $A_{L L}$ in $\vec{p}+\vec{p} \rightarrow$ jet $+X$ at $\sqrt{s}=200 \mathrm{GeV}$ versus jet $p_{T}$ calculated with the polarized toy gluon model (20). Data are from the STAR experiment [6].

(1) The PDF helicity components defined by Fermi-Dirac expressions are the building blocks of the unpolarized and polarized PDFs.

(2) The thermodynamical potentials satisfy a hierarchy relation given by Eq. (10) which imposes specific properties on the distribution functions.

(3) The expressions obtained between quark and antiquarks allow one to relate the behaviors of the ratios $x d\left(x, Q^{2}\right) / x u\left(x, Q^{2}\right)$ and $x \bar{u}\left(x, Q^{2}\right) / x \bar{d}\left(x, Q^{2}\right)$.

(4) A toy model has been defined for the gluon in terms of unpolarized and polarized quarks distributions that produces equivalent results to the original gluon parametrizations but with only one free normalization parameter. In addition this toy model gives for the gluon made with basic fermion helicity components a relation between unpolarized and polarized gluon distributions, which was not the case in the original version of the model.

It is clear that the model is able to explain a large set of unpolarized and polarized experimental deep inelastic scattering data. Of course the predictions which can be made in view of future experiments depend on the present values of the parameters, so it is a challenge for the model to be confirmed by new experiments.

To conclude, the statistical approach not only provides numerical PDF values compatible with experimental data but also gives a coherent model of the quark structure at the fundamental level of helicity distributions.
[1] S. Dulat, T.-J. Hou, J. Gao, M. Guzzi, J. Huston, P. Nadolsky, J. Pumplin, C. Schmidt, D. Stump, and C.-P. Yuan, Phys. Rev. D 93, 033006 (2016).

[2] L. A. Harland-Lang, A. D. Martin, P. Motylinski, and R. S. Thorne, Eur. Phys. J. C 75, 204 (2015).

[3] C. Bourrely, F. Buccella, and J. Soffer, Eur. Phys. J. C 23, 487 (2002).

[4] G. P. Salam and J. Rojo, Comput. Phys. Commun. 180, 120 (2009).
[5] C. Bourrely and J. Soffer, Nucl. Phys. A 941, 307 (2015).

[6] L. Adamczyk et al. (STAR Collaboration), Phys. Rev. Lett. 113, 072301 (2014).

[7] K. Ackerstaff et al. (HERMES Collaboration), Phys. Lett. B 404, 383 (1997).

[8] K. Ackerstaff et al. (HERMES Collaboration), Phys. Lett. B 464, 123 (1999).

[9] A. Airapetian et al. (HERMES Collaboration), Phys. Lett. B 442, 484 (1998). 
[10] X. Zheng et al., Phys. Rev. C 70, 065207 (2004).

[11] X. Zheng et al., Phys. Rev. Lett. 92, 012004 (2004).

[12] C. Bourrely, J. Soffer, and F. Buccella, Eur. Phys. J. C 41, 327 (2005).

[13] M. Salajegheh, H. Khanpour, and S. M. Moosavi Nejad, Phys. Rev. C 96, 065205 (2017).

[14] S. Kuhlmann et al., Phys. Lett. B 476, 291 (2000).

[15] E. A. Hawker, T. C. Awes, M. E. Beddo, C. N. Brown, J. D. Bush, T. A. Carey, T. H. Chang, W. E. Cooper, C. A. Gagliardi, G. T. Garvey et al. (FNAL NuSea Collaboration), Phys. Rev. Lett. 80, 3715 (1998).

[16] J. C. Peng, G. T. Garvey, T. C. Awes, M. E. Beddo, M. L. Brooks, C. N. Brown, J. D. Bush, T. A. Carey, T. H. Chang, W. E. Cooper et al., Phys. Rev. D 58, 092004 (1998).
[17] P. Reimer, The Quest for the Origin of the Proton's Sea, invited talk at DIFFRACTION 2016: International Workshop on Diffraction in High-Energy Physics, Sept. 2-8, 2016, Santa Tecla di Acireale, Italy (unpublished).

[18] C. Bourrely, New Properties of the Parton Statistical Model (Scholar's Press, Saarbrücken, 2016); see also arXiv:1507.03752.

[19] C. Bourrely and J. Soffer, Phys. Lett. B 740, 168 (2015).

[20] H. M. Srivastava et al., Russ. J. Math. Phys. 11, 107 (2011).

[21] D. de Florian, R. Sassot, M. Stratmann, and W. Vogelsang, Phys. Rev. D 80, 034030 (2009).

[22] D. de Florian, R. Sassot, M. Stratmann, and W. Vogelsang, Phys. Rev. Lett. 113, 012001 (2014).

[23] B. I. Abelev et al., Phys. Rev. Lett. 97, 252001 (2006). 